UDK 517.5

\author{
K. V. Pozharska*, O. A. Pozharskyi** \\ * Institute of Mathematics of NAS of Ukraine, \\ Kyiv 01024.E-mail: kate.shvai@gmail.com \\ ** Institute of Mathematics of NAS of Ukraine, \\ Kyiv 01024.E-mail: PozharskyiO@gmail.com
}

\title{
Recovery of continuous functions from their Fourier coefficients known with error ${ }^{1}$
}

Розглядається задача оптимального відновлення функцій за їхніми коефіцієнтами Фур'є, що задані з похибкою. У загальнішій постановці цю задачу для класів гладких і аналітичних функцій, визначених на різних компактних многовидах, розглянуто у класичній статті Г.Г. Магаріл-Ільяєва, К.Ю. Осипенка.

А саме, роботу присвячено відновленню неперервних дійснозначних функцій $y$ однієї змінної з класів $W_{p}^{\psi}, 1 \leq p<\infty$, що задаються в термінах узагальненої гладкості $\psi$ за їхніми коефіцієнтами Фур'є по деякій повній ортонормованій у просторі $L_{2}$ системі $\Phi=\left\{\varphi_{k}\right\}_{k=1}^{\infty}$ неперервних функцій, які збурені шумом.

Вважаємо, що для функції $y$ відомі значення $y_{k}^{\delta}$ її збурених коефіцієнтів Фур'є, причому $y_{k}^{\delta}=y_{k}+\delta \xi_{k}, k=1,2, \ldots$, де $y_{k}-$ відповідні коефіцієнти Фур'є, $\delta \in(0,1)$, а $\xi=\left(\xi_{k}\right)_{k=1}^{\infty}$ - деякий шум. Додатково припускаємо, що функції системи $\Phi$ неперервні і підпорядковані умові $\left\|\varphi_{k}\right\|_{C} \leq C_{1} k^{\beta}, k=1,2, \ldots$, де $C_{1}>0, \beta \geq 0$ - деякі сталі, а $\|\cdot\| C$ норма простору $C$ неперервних на відрізку $[0,1]$ функцій, визначена у стандартний спосіб.

За певних умов щодо параметра $\psi$, встановлено порядкові оцінки похибок наближень функцій із класів

$$
W_{p}^{\psi}=\left\{y \in L_{2}:\|y\|_{W_{p}^{\psi}}^{p}=\sum_{k=1}^{\infty} \psi^{p}(k)\left|y_{k}\right|^{p} \leq 1\right\}, \quad 1 \leq p<\infty,
$$

у метриці простору $C$ за допомогою так званого $\Lambda$-методу підсумовування рядів, що задається трикутними числовими матрицями $\Lambda=\left\{\lambda_{k}^{n}\right\}_{k=1}^{n}, n=n(\delta) \in \mathbb{N}$, iз певними обмеженнями щодо їхніх елементів.

Зазначимо, що у результаті проведених досліджень, нами поширено відомі результати $[7,8]$ на більш широкий спектр класів функцій і при загальніших обмеженнях на рівень шуму, адже охоплено випадок, коли шум є сильнішим, ніж у просторі $l_{2}$ дійсних числових послідовностей, але не є стохастичним.

Ключові слова: Ряди Фур'є, методи регуляризацій,

\footnotetext{
${ }^{1}$ The work was partially financially supported by the grant H2020-MSCA-RISE-2014, project number 645672 (AMMODIT: Approximation Methods for Molecular Modelling and Diagnosis Tools) and the budget program "Support of the development of priority branches of scientific research in 2020" (KPKVK 6541230). Authors are grateful to V.S. Romanyuk for valuable comments, remarks and assistance in preparing the paper.
} 
The problem of optimal recovery is considered for functions from their Fourier coefficients known with error. In a more general statement, this problem for the classes of smooth and atalytic functions defined on various compact manifolds can be found in the classical paper by G.G. Magaril-Il'yaev, K.Y. Osipenko.

Namely, the paper is devoted to the recovery of continuous real-valued functions $y$ of one variable from the classes $W_{p}^{\psi}, 1 \leq p<\infty$, that are defined in terms of generalized smoothness $\psi$ from their Fourier coefficients with respect to some complete orthonormal in the space $L_{2}$ system $\Phi=\left\{\varphi_{k}\right\}_{k=1}^{\infty}$ of continuous functions, that are blurred by noise.

Assume that for function $y$ we know the values $y_{k}^{\delta}$ of their noisy Fourier coefficients, besides $y_{k}^{\delta}=y_{k}+\delta \xi_{k}, k=1,2, \ldots$, where $y_{k}$ are the corresponding Fourier coefficients, $\delta \in(0,1)$, and $\xi=\left(\xi_{k}\right)_{k=1}^{\infty}$ is a noise. Additionally let the functions from the system $\Phi$ are continuous and satisfy the condition $\left\|\varphi_{k}\right\|_{C} \leq C_{1} k^{\beta}, k=1,2, \ldots$, where $C_{1}>0, \beta \geq 0$ are some constants, and $\|\cdot\|_{C}$ is the standart norm of the space $C$ of continuous on the segment $[0,1]$ functions.

Under certain conditions on parameter $\psi$, we obtain order estimates of the approximation errors of functions from the classes

$$
W_{p}^{\psi}=\left\{y \in L_{2}:\|y\|_{W_{p}^{\psi}}^{p}=\sum_{k=1}^{\infty} \psi^{p}(k)\left|y_{k}\right|^{p} \leq 1\right\}, \quad 1 \leq p<\infty,
$$

in metric of the space $C$ by the so-called $\Lambda$-method of series summation that is defined by the number triangular matrix $\Lambda=\left\{\lambda_{k}^{n}\right\}_{k=1}^{n}, n=n(\delta) \in \mathbb{N}$, with some restrictions on its elements.

Note, that we extend the known results $[8,7]$ to a more wide spectrum of the classes of functions and for a more general restrictions on the noise level. In our results a case is considered when the noise is stronger than those in the space $l_{2}$ of real number sequences, but not stochastic.

Key words: Fourier series, methods of regularization, $\Lambda$-methods of summation

MSC2010: PRI 41A10, SEC 46E35, $47 \mathrm{~A} 52$

\section{Introduction}

Let $L_{p}:=L_{p}([0,1]), 1 \leq p<\infty$, be the space of real-valued summable with $p$ th power on the segment $[0,1]$ functions $f:[0,1] \rightarrow \mathbb{R}$ equipped with the norm

$$
\|f\|_{L_{p}}:=\left(\int_{0}^{1}|f(x)|^{p} \mathrm{~d} x\right)^{\frac{1}{p}}, \quad 1 \leq p<\infty ;
$$

$C:=C([0,1])$ be the space of continuous on $[0,1]$ functions $f:[0,1] \rightarrow \mathbb{R}$ with the norm

$$
\|f\|_{C}:=\max _{x \in[0,1]}|f(x)|
$$

$l_{p}, 1 \leq p<\infty$, be the set of sequences $\xi=\left(\xi_{k}\right)_{k=1}^{\infty}$ of real numbers, such that

$$
\|\xi\|_{l_{p}}:=\left(\sum_{k=1}^{\infty}\left|\xi_{k}\right|^{p}\right)^{\frac{1}{p}}<\infty
$$


$l_{\infty}$ be the space of bounded sequences $\xi=\left(\xi_{k}\right)_{k=1}^{\infty}$ of real numbers with the norm

$$
\|\xi\|_{l_{\infty}}:=\sup _{k \in \mathbb{N}}\left|\xi_{k}\right| .
$$

For function $y \in C$ let us consider its Fourier series

$$
\sum_{k=1}^{\infty} y_{k} \varphi_{k}(t)
$$

where

$$
y_{k}:=\left\langle y, \varphi_{k}\right\rangle_{L_{2}}=\int_{0}^{1} y(x) \varphi_{k}(x) \mathrm{d} x
$$

are the Fourier coefficients of the function $y$ with respect to some complete orthonormal in the space $L_{2}$ with the inner product $\langle\cdot, \cdot\rangle_{L_{2}}$ system $\Phi:=\left\{\varphi_{k}\right\}_{k=1}^{\infty}$ of continuous on $[0,1]$ functions.

Further, for $y \in C$, let us know only approximate values $y_{k}^{\delta}$ of its Fourier coefficients $y_{k}$, such that

$$
y_{k}^{\delta}=y_{k}+\delta \xi_{k}, \quad k=1,2, \ldots,
$$

where $\delta \in(0,1)$ and $\xi=\left(\xi_{k}\right)_{k=1}^{\infty}$ is a noise, for which

$$
\|\xi\|_{l_{p}} \leq 1 .
$$

A recovery problem for functions $y$ that belong to some class $F \subset C$ from the set $Y^{\delta, p}(F)$ of noisy Fourier coefficients $y^{\delta, p}:=\left(y_{k}^{\delta}\right)_{k=1}^{\infty}$ of all functions $y \in F$ determined in the indicated above way, consists in determination or choosing a mapping $A: Y^{\delta, p}(F) \rightarrow$ $C$ (method of the recovery) such that the quantity

$$
\varepsilon(\delta)=\Delta\left(F, A, l_{p}, C\right):=\sup _{y \in F} \sup _{\|\xi\|_{p} \leq 1}\left\|y-A y^{\delta, p}\right\|_{C}
$$

tends to zero as $\delta \rightarrow 0$.

The aim of the present paper is to estimate from above the quantity

$$
\Delta\left(W_{p}^{\psi}, \Lambda, l_{p}\right):=\sup _{y \in W_{p}^{\psi}} \sup _{\|\xi\|_{p} \leq 1}\left\|y-\sum_{k=1}^{n} \lambda_{k}^{n}\left(y_{k}+\delta \xi_{k}\right) \varphi_{k}\right\|_{C},
$$

$1 \leq p<\infty, \delta \in(0,1)$, when:

- functions of the system $\Phi=\left\{\varphi_{k}\right\}_{k=1}^{\infty}$ satisfy the condition

$$
\left\|\varphi_{k}\right\|_{C} \leq C_{1} k^{\beta}, \quad k=1,2, \ldots,
$$

with some constants $C_{1}>0, \beta \geq 0$; 


\section{RECOVERY OF CONTINUOUS FUNCTIONS}

- elements $\lambda_{k}^{n}$ of the triangular number matrix $\Lambda:=\left\{\lambda_{k}^{n}\right\}_{k=1}^{n}, n=n(\delta) \in \mathbb{N}$, satisfy the condition

$$
\left|1-\lambda_{k}^{n}\right| \leq C_{2}\left(\frac{k}{n}\right)^{\theta}, \quad 1 \leq k \leq n, n \in \mathbb{N},
$$

where the number $\theta>0$ and $C_{2}$ is a positive constant;

- $W_{p}^{\psi}=\left\{y \in L_{2}:\|y\|_{W_{p}^{\psi}}^{p}=\sum_{k=1}^{\infty} \psi^{p}(k)\left|y_{k}\right|^{p} \leq 1\right\}$, where the sequence $\{\psi(k), k \in \mathbb{N}\}$ belongs to the further defined set $\Psi_{\gamma_{1}, \gamma_{2}}, \quad 0<\gamma_{1}<\gamma_{2}$.

The set of systems for which the condition (1.2) holds is denoted by $K^{\beta}$. Note, that to the set $K^{\beta}$ with $\beta=0$ belongs, in particular, the trigonometric system $\mathcal{T}=$ $\left\{e^{2 \pi i k x}\right\}_{k \in \mathbb{Z}}$, and also the system of Legendre polynomials (with $\beta=\frac{1}{2}$ ). As for the condition (1.3), here, for example, for the Fejér method $\lambda_{k}^{n}=1-k / n$ and hence the order is $\theta=1$. For the Zygmund method we have $\lambda_{k}^{n}=1-(k / n)^{s}, s>0$, and therefore $\theta=s$. Note also, that in the considered case, as a part of the described above recovery problem,

$$
A=T_{n}^{\Lambda}\left(y^{\delta}\right)(t):=\sum_{k=1}^{n} \lambda_{k}^{n} y_{k}^{\delta} \varphi_{k}(t),
$$

is the so-called $\Lambda$-method (of order $\theta$ ) with respect to the system $\Phi$. Besides, further we show that under certain conditions the embedding $W_{p}^{\psi} \subset C$ is true.

The main result of the paper is the following order estimate

$$
\Delta\left(W_{p}^{\psi}, \Lambda, l_{p}\right) \ll \delta\left(\psi^{-1}([1 / \delta])\right)^{\beta+1-\frac{1}{p}} \asymp \frac{1}{\psi(n)} n^{\beta+1-\frac{1}{p}},
$$

that is true for $n \asymp \psi^{-1}([1 / \delta])$ under certain conditions on the function $\psi$ (that depend on $p, \beta$ and $\theta$ ).

Here and below, the notation $A \asymp B$ for a positive number sequence $A=\left(A_{n}\right)_{n=1}^{\infty}$ and function $B=B(\delta), \delta \in(0,1)$, that may depend on some set of parameters, means that for all admissible values of this parameters under certain connection between $n \in \mathbb{N}$ and $\delta \in(0,1)$ the relations $c_{1} B \leq A \leq c_{2} B$ are true with certain positive quantities $c_{1}$ and $c_{2}$ that do not depend on $n \in \mathbb{N}$ and $\delta \in(0,1)$. We also use symbols $\ll$ and $\gg$, i.e., $A \ll B \quad(A \gg B)$, if $A \leq c B(B \leq c A)$ for some $c>0$ that does not depend on $n \in \mathbb{N}$ and $\delta \in(0,1)$. In the case of two positive number sequences $A=\left(A_{n}\right)_{n=1}^{\infty}$ and $D=\left(D_{n}\right)_{n=1}^{\infty}$, under the indicated above conditions we write $A \asymp D$, $A \ll D$ and $A \gg D$ (the constants $c_{i}$ in corresponding inequalities do not depend on the parameter $n \in \mathbb{N}$ ).

The recovery problem in a sense of estimating the quantities $\Delta\left(F, A, l_{p}, C\right)$ belongs to so-called "ill-posed problems". It is so because in general the corresponding approximation error is not stable for small (in the metric $l_{p}$ ) changes of the Fourier coefficients, when measuring the error in the metric $C$ (see [14, Example 3, p. 19; Ch. VII], [4, P. 452-459]). 
Quite complete information on a general problem statement for the optimal recovery in normed spaces, as well as corresponding results for the classes of smooth and analytic functions defined on various compact manifolds can be found in [6]. The related problems are investigated also in $[2,3]$ were one can find further bibliography.

In the paper by A.N. Tikhonov [12] a method of series summation was suggested that is stable for small in the metric $l_{2}$ changes of the coefficients $y_{k}, k=1,2, \ldots$, based on the idea of regularization (general ideas of regularization were formulated earlier in the paper [13]). This method consists in (see [14, Ch. VII]) approximating function $y$ by series of the form

$$
T^{\alpha}\left(y^{\delta}\right)(t)=\sum_{k=1}^{\infty} \frac{1}{1+\alpha a_{k}} y_{k}^{\delta} \varphi_{k}(t)
$$

where $\alpha$ is a regularizing parameter that depends on the error of input data, i.e., $\alpha=\alpha(\delta)$, and $\left(a_{k}\right)_{k=1}^{\infty}$ is a sequence of positive numbers such that as $k \rightarrow \infty$ increases not slower than $k^{2+\varepsilon}, \varepsilon>0$. In the paper by P. Mathe, S.V. Pereverzyev [7] a $\Lambda$-method

$$
T_{n}^{\Lambda}\left(y^{\delta}\right)(t)=\sum_{k=1}^{n} \lambda_{k}^{n} y_{k}^{\delta} \varphi_{k}(t)
$$

of series summation was considered for the first time as a recovery method namely in studying "ill-posed" problems. Note, that this method is widely used also in solving direct ("well-posed") problems of function recovery (see, e.g., [5, §2.2]). As well, note that the use of $\Lambda$-methods is quite natural and important in the classical approximation theory, in particular, in investigations connected with various problems of function approximation (see, e.g., [9, Ch. 2.2]).

Now we mention some known results that are directly related to the result of our research. So, in the cited above paper [7] the estimates are obtained of the quantities $\Delta\left(W_{2}^{\mu}, \Lambda, l_{2}\right)$ for the function class

$$
W_{2}^{\mu}=\left\{y \in L_{2}: \quad\|y\|_{W_{2}^{\mu}}^{2}=\sum_{k=1}^{\infty} k^{2 \mu}\left|y_{k}\right|^{2} \leq 1\right\},
$$

where $y_{k}, k=1,2, \ldots$, are the Fourier coefficients of function $y$ with respect to the orthonormal system $\Phi$ that belongs to the set $K^{\beta}, \beta+1 / 2<\mu \leq \theta$.

In [8] K. Sharipov extended the result from [7] on the spectrum of classes $W_{2}^{\psi}$ of continuous functions

$$
W_{2}^{\psi}=\left\{y \in L_{2}: \quad\|y\|_{W_{2}^{\psi}}^{2}=\sum_{k=1}^{\infty} \psi^{2}(k)\left|y_{k}\right|^{2} \leq 1\right\},
$$

where $\{\psi(k), k \in \mathbb{N}\}$ is an arbitrary monotonically increasing sequence such that the order of its growth is between $k^{\beta+1 / 2}$ and $k^{\theta}, \beta+1 / 2<\theta$.

In the problem statement of this paper, firstly, the case is covered when the noise $\xi$ is stronger than in the space $l_{2}$ (in view of $\|\xi\|_{l_{2}} \leq\|\xi\|_{l_{p}}$ if $1 \leq p \leq 2$ ), but still not 
stochastic. Secondly, a quite wide spectrum of the classes $W_{p}^{\psi}, 1 \leq p<\infty$, is considered of functions to recover. As to the classes $W_{p}^{\psi}$, we additionally note that for fixed system $\Phi$ and function $\psi$ the following embedding holds: $W_{p}^{\psi} \subseteq W_{q}^{\psi}, 1 \leq p<q<\infty$.

We should also mention, that in the paper [11] the classes (and spaces) $S^{p}$ were introduced with non-symmetric metric that in partial case coincide with the classes $W_{p}^{\psi}$. There (see also [10]) the estimates are obtained for the best approximations and Kolmogorov widths of $q$-ellipsoids in this spaces. Direct and inverse theorems of approximation theory in the Orlicz spaces with the so-called Luxemburg norm are obtained in the paper [1] on the classes of periodic functions from the space

$$
S_{\mathbf{p}, \mu}:=\left\{f \in L: \quad \sum_{k \in \mathbb{Z}} \mu_{k}|\widehat{f}(k)|^{p_{k}}<\infty\right\},
$$

where $\mathbf{p}=\left\{p_{k}\right\}_{k \in \mathbb{Z}}, 1 \leq p_{k} \leq K, K>0, \mu=\left\{\mu_{k}\right\}_{k \in \mathbb{Z}}, \mu_{k}>0$, and $\widehat{f}(k)$ are the Fourier coefficients of function $f$ with respect to the trigonometric system.

In what follows, by $y^{-1}$ we denote an inverse to $y$ function. The symbol $[a]$ stands for an integer part of the number $a \in \mathbb{R}$.

\section{Main results}

By $\Psi_{\gamma_{1}, \gamma_{2}}, \quad 0<\gamma_{1}<\gamma_{2}$, we denote the set of continuous, positive and strictly increasing on $[1, \infty)$ real-valued functions $\psi$ that satisfy the conditions:

1) for some $\gamma, \gamma_{1}<\gamma<\gamma_{2}$, the function $\phi_{-}(\tau):=\frac{\tau^{\gamma}}{\psi(\tau)}$ does not increase for $\tau \geq 1$, and $\phi_{-}(\tau) \rightarrow 0$ as $\tau \rightarrow \infty$;

2) the function $\phi_{+}(\tau):=\frac{\tau^{\gamma_{2}}}{\psi(\tau)}$ does not decrease for $\tau \geq 1$.

To the set $\Psi_{\gamma_{1}, \gamma_{2}}$ belong, in particular, power functions $\psi(\tau)=\tau^{\alpha_{1}}$ with $\gamma_{1}<\alpha_{1} \leq \gamma_{2}$, and also functions of the form $\psi(\tau)=\tau^{\alpha_{1}}\left(\log ^{+}(\tau)\right)^{\alpha_{2}}, \gamma_{1}<\alpha_{1}<\gamma_{2}, \alpha_{2} \in \mathbb{R}$ or $\psi(\tau)=\tau^{\gamma_{2}}\left(\log ^{+}(\tau)\right)^{\alpha}, \alpha<0$, where $\log ^{+}(\tau)=\max \{1, \log (\tau)\}$, and the symbol $\log$ denotes a logarithm with arbitrary base $a>0, \quad a \neq 1$.

The following statement holds.

Theorem 1. Let $1 \leq p \leq 2, \quad \psi \in \Psi_{\beta+\frac{1}{2}, \theta}$, or $2<p<\infty, \quad \psi \in \Psi_{\beta+1, \theta}$ and $T_{n}^{\Lambda}\left(y^{\delta}\right)$ is a method of recovery defined by the formula (1.4). Then for $n \asymp \psi^{-1}([1 / \delta])$ we have the estimate

$$
\Delta\left(W_{p}^{\psi}, \Lambda, l_{p}\right) \ll \frac{1}{\psi(n)} n^{\beta+1-\frac{1}{p}},
$$

or writing the same in terms of the parameter $\delta$ :

$$
\Delta\left(W_{p}^{\psi}, \Lambda, l_{p}\right) \ll \delta\left(\psi^{-1}([1 / \delta])\right)^{\beta+1-\frac{1}{p}} .
$$


Proof. First note, that under the conditions of Theorem 1 the set $W_{p}^{\psi}$ consists of continuous on the segment $[0,1]$ functions, and for any $y \in W_{p}^{\psi}$ its Fourier series $\sum_{k=1}^{\infty} y_{k} \varphi_{k}(t)$ with respect to the system $\Phi$ converges uniformly on $[0,1]$ to $y$, because

$$
\left\|\sum_{k=n+1}^{\infty} y_{k} \varphi_{k}\right\|_{C} \rightarrow 0 \quad \text { as } n \rightarrow \infty
$$

(see further estimate of the term $I_{2}$ from the relation (2.1)).

Therefore, we can write

$$
\begin{aligned}
\left\|y-T_{n}^{\Lambda}\left(y^{\delta}\right)\right\|_{C} \leq & \left\|\sum_{k=1}^{n}\left(1-\lambda_{k}^{n}\right) y_{k} \varphi_{k}\right\|_{C}+\left\|\sum_{k=n+1}^{\infty} y_{k} \varphi_{k}\right\|_{C} \\
& +\delta\left\|\sum_{k=1}^{n} \lambda_{k}^{n} \xi_{k} \varphi_{k}\right\|_{C}=: I_{1}+I_{2}+I_{3} .
\end{aligned}
$$

Let us estimate first the term $I_{1}$ from (2.1). Taking into account the condition (1.3) and norm properties, we obtain

$$
\begin{aligned}
I_{1} & =\left\|\sum_{k=1}^{n}\left(1-\lambda_{k}^{n}\right) y_{k} \varphi_{k}\right\|_{C} \leq C_{2} \sum_{k=1}^{n}\left(\frac{k}{n}\right)^{\theta}\left|y_{k}\right|\left\|\varphi_{k}\right\|_{C} \\
& =C_{2} \sum_{k=1}^{n}\left(\frac{k}{n}\right)^{\theta} \psi(k)\left|y_{k}\right| \frac{1}{\psi(k)}\left\|\varphi_{k}\right\|_{C} .
\end{aligned}
$$

Now note the following. In further speculations, in particular, when using the Hölder's inequality for number sequences, an important thing in the form of writi$\mathrm{ng}$ is that $p \neq 1$. Nevertheless, this speculations as well as their result remain true also for $p=1$ after their corresponding correction with respect to the definition of norm in the space $l_{\infty}$.

Therefore, applying the Hölder's inequality to the right-hand side of (2.2) for $p \neq 1$, $\frac{1}{p}+\frac{1}{p^{\prime}}=1$, we get

$$
\begin{aligned}
I_{1} & \leq C_{2}\left(\sum_{k=1}^{n}\left(\psi(k)\left|y_{k}\right|\right)^{p}\right)^{\frac{1}{p}}\left(\sum_{k=1}^{n}\left(\left(\frac{k}{n}\right)^{\theta} \frac{1}{\psi(k)}\left\|\varphi_{k}\right\|_{C}\right)^{p^{\prime}}\right)^{\frac{1}{p^{\prime}}} \\
& \leq C_{2}\|y\|_{W_{p}^{\psi}}\left(\sum_{k=1}^{n}\left(\frac{k^{\theta}}{\psi(k)} \frac{\left\|\varphi_{k}\right\|_{C}}{n^{\theta}}\right)^{p^{\prime}}\right)^{\frac{1}{p^{\prime}}} .
\end{aligned}
$$

Now, taking into account, that the function $\psi$ satisfies condition 2) in the definition 
of the set $\Psi_{\gamma_{1}, \gamma_{2}}$ with $\gamma_{2}=\theta$, and in view of (1.2) from (2.3) we have

$$
\begin{aligned}
I_{1} & \leq C_{2} \frac{n^{\theta}}{\psi(n) n^{\theta}}\left(\sum_{k=1}^{n}\left\|\varphi_{k}\right\|_{C}^{p^{\prime}}\right)^{\frac{1}{p^{\prime}}} \leq C_{1} C_{2} \frac{1}{\psi(n)}\left(\sum_{k=1}^{n} k^{\beta p^{\prime}}\right)^{\frac{1}{p^{\prime}}} \\
& \leq C_{1} C_{2} \frac{1}{\psi(n)}\left(n^{\beta p^{\prime}} n\right)^{\frac{1}{p^{\prime}}}=C_{3} \frac{1}{\psi(n)} n^{\beta+1-\frac{1}{p}} .
\end{aligned}
$$

The same estimate holds also for $p=1$.

Further we move to estimation of the term $I_{2}$ from the right-hand side of (2.1). First we write

$$
\begin{aligned}
I_{2} & =\left\|\sum_{k=n+1}^{\infty} y_{k} \varphi_{k}\right\|_{C}=\left\|\sum_{k=n+1}^{\infty} \psi(k) y_{k} \frac{1}{\psi(k)} \varphi_{k}\right\|_{C} \\
& \leq\left(\sum_{k=n+1}^{\infty}\left(\psi(k)\left|y_{k}\right|\right)^{p}\right)^{\frac{1}{p}}\left(\sum_{k=n+1}^{\infty}\left(\frac{\left\|\varphi_{k}\right\|_{C}}{\psi(k)}\right)^{p^{\prime}}\right)^{\frac{1}{p^{\prime}}} .
\end{aligned}
$$

In what follows, we split estimation of the right-hand side of (2.5) into two cases: when $1<p \leq 2$ and $2<p<\infty$.

Let $1<p \leq 2$, then from $(2.5)$ we obtain

$$
I_{2} \leq\|y\|_{W_{p}^{\psi}}\left(\sum_{k=n+1}^{\infty}\left(\frac{\left\|\varphi_{k}\right\|_{C}}{k^{\beta+\frac{1}{2}+\varepsilon}} \frac{k^{\beta+\frac{1}{2}+\varepsilon}}{\psi(k)}\right)^{p^{\prime}}\right)^{\frac{1}{p^{\prime}}}
$$

(here $\varepsilon>0$ is arbitrary).

By the conditions of Theorem 1, the function $\psi \in \Psi_{\beta+\frac{1}{2}, \theta}$, therefore for sufficiently small $\varepsilon>0$ taking into account that $\|y\|_{W_{p}^{\psi}} \leq 1$ and the condition (1.2), from (2.6) we get

$$
I_{2} \leq C_{1} \frac{n^{\beta+\frac{1}{2}+\varepsilon}}{\psi(n)}\left(\sum_{k=n+1}^{\infty} k^{-\left(\frac{1}{2}+\varepsilon\right) p^{\prime}}\right)^{\frac{1}{p^{\prime}}} .
$$

In view of $1<p \leq 2$, it holds $\left(\frac{1}{2}+\varepsilon\right) p^{\prime}>1$, and therefore using the inequality

$$
\sum_{k=n+1}^{\infty} f(k) \leq \int_{n}^{\infty} f(x) \mathrm{d} x
$$

that holds for arbitrary positive continuous and decreasing on $[n, \infty)$ function $f$, such that the series $\sum_{k=1}^{\infty} f(k)$ is convergent, we first have

$$
\begin{aligned}
\sum_{k=n+1}^{\infty} k^{-\left(\frac{1}{2}+\varepsilon\right) p^{\prime}} & \leq \int_{n}^{\infty} x^{-\left(\frac{1}{2}+\varepsilon\right) p^{\prime}} \mathrm{d} x \\
& =\left.\frac{x^{-\left(\frac{1}{2}+\varepsilon\right) p^{\prime}+1}}{-\left(\frac{1}{2}+\varepsilon\right) p^{\prime}+1}\right|_{n} ^{\infty}=\frac{n^{-\left(\frac{1}{2}+\varepsilon\right) p^{\prime}+1}}{\left(\frac{1}{2}+\varepsilon\right) p^{\prime}-1}
\end{aligned}
$$


Now, combining (2.8) with (2.7), we get

$$
\begin{aligned}
I_{2} & \leq C_{1} \frac{n^{\beta+\frac{1}{2}+\varepsilon}}{\psi(n)}\left(\frac{n^{-\left(\frac{1}{2}+\varepsilon\right) p^{\prime}+1}}{\left(\frac{1}{2}+\varepsilon\right) p^{\prime}-1}\right)^{\frac{1}{p^{\prime}}} \\
& =C_{1}\left(\frac{1}{\left(\frac{1}{2}+\varepsilon\right) p^{\prime}-1}\right)^{\frac{1}{p^{\prime}}} \frac{n^{\beta+\frac{1}{2}+\varepsilon}}{\psi(n)} n^{\frac{1}{2}-\frac{1}{p}-\varepsilon} \\
& =C_{1}\left(\frac{1}{\left(\frac{1}{2}+\varepsilon\right) p^{\prime}-1}\right)^{\frac{1}{p^{\prime}}} \frac{n^{\beta+1-\frac{1}{p}}}{\psi(n)} .
\end{aligned}
$$

The same estimate, obviously, holds also for $p=1$.

Assume now, that $2<p<\infty$. Here, as in the previous case, we take into account the condition $\psi \in \Psi_{\beta+1, \theta}$, and for sufficiently small $\varepsilon>0$ from (2.5) we obtain

$$
I_{2} \leq C_{1} \frac{n^{\beta+1+\varepsilon}}{\psi(n)}\left(\sum_{k=n+1}^{\infty} k^{-(1+\varepsilon) p^{\prime}}\right)^{\frac{1}{p^{\prime}}}
$$

and since $1<p^{\prime}<2$ then $(1+\varepsilon) p^{\prime}>1$, and therefore

$$
I_{2} \leq C_{1}\left(\frac{1}{(1+\varepsilon) p^{\prime}-1}\right)^{\frac{1}{p^{\prime}}} \frac{n^{\beta+1-\frac{1}{p}}}{\psi(n)} .
$$

Form (2.9) and (2.10) we conclude that

$$
I_{2} \leq C_{4}(p) \frac{n^{\beta+1-\frac{1}{p}}}{\psi(n)}, \quad 1 \leq p<\infty .
$$

It remains to estimate the third term from the right-hand side of (2.1). Note, that the condition (1.3) yields uniform boundness of the elements $\lambda_{k}^{n}, 1 \leq k \leq n, n \in \mathbb{N}$, of the matrix $\Lambda$. So, we can write

$$
I_{3}=\delta\left\|\sum_{k=1}^{n} \lambda_{k}^{n} \xi_{k} \varphi_{k}\right\|_{C} \leq C_{5} \delta \sum_{k=1}^{n}\left|\xi_{k}\right|\left\|\varphi_{k}\right\|_{C} .
$$

Using the Hölder's inequality to the right-hand side of $(2.12)$ for $\frac{1}{p}+\frac{1}{p^{\prime}}=1, \quad p \neq 1$, and then taking into account the conditions (1.1), (1.2), we get

$$
\begin{aligned}
I_{3} & \leq C_{5} \delta\|\xi\|_{l_{p}}\left(\sum_{k=1}^{n}\left\|\varphi_{k}\right\|_{C}^{p^{\prime}}\right)^{\frac{1}{p^{\prime}}} \\
& \leq C_{1} C_{5} \delta\left(\sum_{k=1}^{n} k^{\beta p^{\prime}}\right)^{\frac{1}{p^{\prime}}} \leq C_{6} \delta n^{\beta+1-\frac{1}{p}} .
\end{aligned}
$$




\section{RECOVERY OF CONTINUOUS FUNCTIONS}

By similar speculations we derive to the same estimate also in the case $p=1$.

Further, combining the initial inequality (2.1) with the estimates (2.4), (2.11) and (2.13), we obtain

$$
\begin{aligned}
\left\|y-T_{n}^{\Lambda}\left(y^{\delta}\right)\right\|_{C} & \leq n^{\beta+1-\frac{1}{p}}\left(\frac{C_{3}+C_{4}(p)}{\psi(n)}+C_{6} \delta\right) \\
& \leq C_{7}(p) n^{\beta+1-\frac{1}{p}}\left(\frac{1}{\psi(n)}+\delta\right) .
\end{aligned}
$$

Further, for $n \in \mathbb{N}$ and $\delta \in(0,1)$ such that $1 / \psi(n) \asymp \delta$ we can write

$$
\Delta\left(W_{p}^{\psi}, l_{p}\right) \ll \frac{1}{\psi(n)} n^{\beta+1-\frac{1}{p}} .
$$

In view of the conditions $\psi \in \Psi_{\beta+\frac{1}{2}, \theta}, 1 \leq p \leq 2$ and $\psi \in \Psi_{\beta+1, \theta}, 2<p<\infty$, we can easily verify that $n \asymp \psi^{-1}([1 / \delta])$. Hence, (2.14) yields

$$
\left\|y-T_{n}^{\Lambda}\left(y^{\delta}\right)\right\|_{C} \ll \delta\left(\psi^{-1}([1 / \delta])\right)^{\beta+1-\frac{1}{p}} .
$$

Theorem 1 is proved.

Remark 1 . Theorem 1 in the case $p=2$, i.e., estimates of the quantity $\Delta\left(W_{2}^{\psi}, \Lambda, l_{2}\right)$, is one of the results from the paper [8]. In particular, for $\psi(k)=k^{\mu}$ the estimates of $\Delta\left(W_{2}^{\mu}, \Lambda, l_{2}\right)$ were obtained in [7]. In this case $\psi^{-1}(k)=k^{1 / \mu}$ and for $\beta+1 / 2<\mu \leq \theta$, $n \asymp \delta^{-1 / \mu}$ we have

$$
\Delta\left(W_{2}^{\mu}, \Lambda, l_{2}\right) \ll \delta^{\frac{\mu-\beta-\frac{1}{2}}{\mu}} .
$$

\section{References}

1. Abdullayev F., Chaichenko S., Imash Kyzy M., Shidlich A.: Direct and inverse approximation theorems in the weighted Orlicz-type spaces with a variable exponent. Turkish J. Math. 44 (1) (2020), 284-299. doi: 10.3906/mat-1911-3

2. Babenko V.F., Gun'ko M.S.: On the optimal reconstruction of the convolution of $n$ functions according to the linear information. Ukr. Mat. J. 68 (5) (2016), 653-660. doi: 10.1007/s11253-016-1248-8

3. Gunko M.S., Babenko V.F., Parfinovych N.V.: Optimal recovery of elements from Hilbert space and their scalar products by Fourier coefficients known with error. Ukr. Mat. Zh. 72 (6) (2020), 736-750. doi: 10.37863/umzh.v72i6.1107

4. Il'in V.A., Poznyak E.G.: Foundations of mathematical analysis. Part II. A course in higher mathematics and mathematical physics. Fourth, revised and supplemented edition. Moscow, FIZMATLIT, (2002). (in Russian)

5. Korneichuk N.P.: Exact constants in approximation theory. Moscow, Nauka, (1987). (in Russian)

6. Magaril-Il'yaev G.G., Osipenko K.Y.: Optimal recovery of functions and their derivatives from Fourier coefficients prescribed with an error. SB MATH 193 (3) (2002), 387-407. doi : $10.1070 /$ SM2002v193n03ABEH000637 
7. Mathe P., Pereverzev S.V.: Stable summation of orthogonal series with noisy coefficients. J. Approx. Theory 118 (2002), 66-80. doi: 10.1006/jath.2002.3710

8. Sharipov K: On the recovery of continuous functions from noisy Fourier coefficients. Comput. Methods in Appl. Math. 11 (1) (2011), 75-82. doi : 10.2478/cmam-2011-0004

9. Stepanets A.I: Classification and approximation of periodic functions. Kyiv, Nauk. Dumka, (1987). (in Russian)

10. Stepanets A.I.: Best approximations of $q$-ellipsoids in spaces $S_{\varphi}^{p, \mu}$. Ukr. Math. J. 56 (2004), 1646-1652. doi: 10.1007/s11253-005-0140-8

11. Stepanets A.I., Rukasov V.I.: Spaces $S^{p}$ with nonsymmetric metric. Ukr. Math. J. 55 (2003), 322-338. doi: 10.1023/A:1025472514408

12. Tihonov A.N.: On stable summability methods for Fourier series. Dokl. Akad. Nauk SSSR 156 (2) (1964), 268-271. (in Russian)

13. Tihonov A.N.: On the solution of ill-posed problems and the method of regularization. Dokl. Akad. Nauk SSSR 151 (3) (1963), 501-504. (in Russian)

14. Tihonov A.N., Arsenin V.Ja.: Methods for the solution of ill-posed problems. Second edition, revised and supplemented. Moscow, Nauka, (1979). (in Russian)

Received: 03.10.2020. Accepted: 14.11.2020 